Submitted to The Astrophysical Journal

\title{
MAGNETIC INVERSION AS A MECHANISM FOR THE SPECTRAL TRANSITION OF BLACK HOLE BINARIES
}

\author{
Igor V. Igumenshchev \\ Laboratory for Laser Energetics, University of Rochester \\ 250 East River Road, Rochester, NY 14623 \\ iigu@lle.rochester.edu
}

\begin{abstract}
A mechanism for the transition between low/hard, high/soft, and steep power law (SPL) spectral states in black hole X-ray binaries is proposed. The low/hard state is explained by the development of a magnetically arrested accretion disk attributable to the accumulation of a vertical magnetic field in a central bundle. This disk forms powerful jets and consists of thin spiral accretion streams of a dense optically thick plasma surrounded by hot, magnetized, optically thin corona, which emits most of the energy in hard X-rays. State transition occurs because of the quasi-periodic or random inversion of poloidal magnetic fields in the accretion flow supplied by the secondary star. The inward advection of the inverted field results in a temporal disappearance of the central bundle caused by the annihilation of the opposed fields and restoration of the optically thick disk in the innermost region. This disk represents the high/soft state. The SPL state develops at the period of intensive field annihilation and precedes the high/soft state. The continuous supply of the inverted field leads to a new low/hard state because of the formation of another magnetically arrested disk.
\end{abstract}

Subject headings: accretion, accretion disks — black hole physics — ISM: jets and outflows - MHD — X-rays: binaries

\section{Introduction}

Accretion disks orbiting black holes (BHs) in X-ray binary systems demonstrate a complicated time-dependent behavior, which is typically described as the quasi-periodic transition between three emission states: the low/hard, high/soft, and steep power law (SPL, or 
otherwise, very high) states (Remillard \& McClintok 2006, hereafter RM06; Done, Gierliński, \& Kubota 2007). The low/hard state is characterized by a strong hard X-ray emission component in the $2-20 \mathrm{keV}$ range, which takes $\gtrsim 80 \%$ of the total flux. A weak, but sizable, thermal component from a cold dense plasma and quasi-periodic oscillations (QPOs) in Xrays may be present or absent. This state is associated with the formation of quasi-steady radio jets (Gallo, Fender, \& Pooley 2003). The high/soft state shows the dominant thermal component, which is generally consistent with theoretical predictions from the "standard" optically thick accretion disk model (Shakura \& Sunyaev 1973). A hard X-ray component is usually present in this state, but is limited to $<25 \%$ of the total flux. The SPL state shares some common properties with the high/soft state, such as the thermal component. However, the SPL state is clearly distinguished by the strength of its power law component and association with high-frequency QPOs (RM06).

While the high/soft state is reasonably well understood theoretically (e.g., Kubota et al. 2005), the nature of the low/hard and SPL states is still a matter of debate. Basically, two classes of phenomenological models were proposed to explain the low/hard state: "truncateddisk" and "corona-and-disk" models. The truncated-disk model assumes that an optically thick disk is truncated at some inner radius $R_{\mathrm{tr}}$ and the central region, $R<R_{\mathrm{tr}}$, is filled with a hot (about the virial temperature), optically thin plasma, which produces hard X-rays (e.g., Zdziarski \& Gierliński 2004). Some studies postulate advection-dominated accretion flows (ADAFs, see Narayan \& Yi 1994; Abramowicz et al. 1995) or other types of hot accretion disk solutions (Shapiro, Lightman, \& Eardley 1976; Blandford \& Begelman 1999; Narayan, Igumenshchev, \& Abramowicz 2000), as sources of this hot plasma (Poutanen, Krolik \& Ryde 1997; Esin, McClintock, \& Narayan 1997; Esin et al. 1998, 2001; D'Angelo et al. 2008). Although such studies can provide excellent fits to the combined optical, UV, and X-ray data, the nature of accretion flows in the central region of luminous sources (with $L \gtrsim 10^{-3} L_{\mathrm{Edd}}$, where $L_{\mathrm{Edd}}$ is the Eddington luminosity) is unexplained. Mechanisms that produce truncated disks have been discussed by Honma (1996) and Manmoto \& Kato (2000), who assumed a radial conductive energy transport that leads to an evaporation of thin disks, and by Meyer, Liu, \& Meyer-Hofmeister (2000), Spruit \& Deufel (2002), and Dullemond \& Spruit (2005), who considered a vertical evaporation process.

In the corona-and-disk models, the optically thick disk remains untruncated and the hard X-rays are generated in a hot, patchy disk corona because of inverse Compton scattering of soft photons that come from the underlying disk (Liang \& Price 1977; Galeev, Rosner, \& Vaiana 1979; Haardt, Maraschi, \& Ghisellini 1994). To reasonably describe observations, the hot corona should dissipate (probably in magnetic flares) a significant fraction ( $\gtrsim 50 \%$ ) of the binding energy of the accretion mass. It is assumed that this energy is transported from disk to corona by a magnetic field, but the exact mechanism is unknown (Merloni \& 
Fabian 2001; Uzdensky \& Goodman 2008).

Both truncated-disk and corona-and-disk models have advantages and disadvantages to explain the observed data (see Done et al. 2007). To overcome the disadvantages, several more sophisticated models were developed, some of which included the elements of both classes of models described above (Taam et al. 2008) and others considered jets that emit in radio and X-rays (Markoff, Falke, \& Fender 2001; Ferreira et al. 2006). The transition between emission states in the truncated-disk models is assumed by means of shifting the truncation radius $R_{\mathrm{tr}}$ to or out of the $\mathrm{BH}$ (e.g., Petrucci et al. 2006). The parameter, or a set of parameters, that triggers the transition is unknown. For example, the triggering mechanism's dependence on the luminosity, or accretion rate, is ruled out by observations that show that many sources can have about same total luminosity at different states (Done \& Gierliński 2003).

This letter proposes a detailed mechanism for spectral variability of BH X-ray binaries. The mechanism uses a recently found dynamical model of magnetized accretion disks (Igumenshchev 2008, hereafter I08) to explain the low/hard and SPL states, and assumes that an inversion of vertical magnetic fields in the disks triggers the state transition. Similar magnetic inversion mechanisms were considered in recent studies of BH X-ray transients and active galactic nuclei (Livio, Pringle, \& King 2003; Tagger et al. 2004) and discussed by Igumenshchev, Narayan, \& Abramowicz (2003, hereafter INA03) in a more general context. The present study specifically focuses on the structure and dynamics of accretion disks that experience magnetic inversion. Details of the model of the low/hard state and its possible observational implications are discussed in Sec. II. Section III describes the transition mechanism, presents supporting simulation results, and briefly discusses the nature of the SPL state. The letter is summarized in Sec. IV.

\section{Model for the Low/Hard State}

The key ingredient of the model is a bipolar magnetic field that is supplied with accretion flows at the outer boundary. Such flows were modeled by 108 using combined 2D/3D numerical MHD simulations. The simulations assume a permanent injection of mass and poloidal magnetic field into a slender equatorial torus located at $R_{\text {inj }}$ near the outer boundary $R_{\text {out }}=220 R_{g}$, where $R_{g}=2 G M / c^{2}$ and $M$ is the BH mass. The injected mass and field form a magnetized accretion disk that carries the vertical magnetic field inward. At the inner numerical boundary, $R_{\mathrm{in}}=2 R_{\mathrm{g}}$, the mass is absorbed by the $\mathrm{BH}$, but the vertical field, which cannot be absorbed, is accumulated in a central bundle. When the field $B_{0}$ in 
the bundle approaches the equipartition level,

$$
\frac{B_{0}^{2}}{8 \pi} \sim \rho \frac{G M}{R_{\mathrm{g}}},
$$

where $\rho$ is the density, the accretion flow is arrested by the field. Further accumulation of the field results in the growth of the outer radius of the arrested region, $R_{\mathrm{m}}$. This type of accretion flow was anticipated in previous theoretical and numerical studies (BisnovatyiKogan \& Ruzmaikin 1974, 1976; INA03; Narayan, Igumenshchev, \& Abramowicz 2003) and was called the magnetically arrested disk. Most of the volume in such a disk is filled with a hot (with temperature $T \sim G M m_{\mathrm{p}} / R$, where $m_{\mathrm{p}}$ is the proton mass), highly magnetized (with plasma $\beta \ll 1$ ), low-density plasma. The accretion disk interacts with this plasma at $R_{\mathrm{m}}$ and forms geometrically thin and dense streams, which accrete into the $\mathrm{BH}$ on spiral trajectories, flowing around low-density "magnetic islands" (see Figs. 4 and 6 in I08). The flow pattern is highly variable because of the development of Rayleigh-Taylor and Kelvin-Helmholtz instabilities. The infall velocity of the dense plasma in streams is a large fraction $(\sim 0.5)$ of the free-fall velocity, which is why the streams are geometrically thin and occupy only a small fraction of the volume in a magnetically arrested disk. The high infall velocity also indicates that there is an efficient "braking" of the rotating flow by means of the vertical field. This braking results in the transfer of the rotational energy of the flow to the field energy and the release of the latter energy in the form of bipolar Poynting jets. The simulations of I08 demonstrated that $\sim 1 \%$ of $\dot{M} c^{2}$, where $\dot{M}$ is the mass accretion rate, can go into the jets in the case of magnetically arrested disks orbiting non-rotating BHs. Note that the jet power can be substantially enhanced in the case of fast rotating BHs with properly aligned spins because of the "ergospheric disk" mechanism (Punsly \& Coroniti 1990; Punsly, Hirose, \& Igumenshchev 2009).

The magnetically arrested disk model is a promising candidate to explain BH binaries in the low/hard state because of its specific properties. This model can have an outer optically thick disk that is naturally truncated at $R_{\mathrm{tr}}=R_{\mathrm{m}}$, similar to what is postulated in the phenomenological truncated-disk model. Inside $R_{\mathrm{m}}$, a soft X-ray emission from the disk is suppressed because of fast accretion of the mass in spiral streams, which an optically thick medium cannot efficiently radiate because of the long Thomson-scattering diffusion time of photons in comparison with the accretion time (I08). Instead, the binding energy of the accretion flow is transformed into the energy of torsional MHD waves that feed the Poynting jets and heat the low-density plasma, which surrounds the dense accretion streams, via magnetic flares. This plasma can emit hard X-rays via bremsstrahlung and Compton scattering of soft photons from the dense streams. Such emission properties plus the presence of the dense plasma in the vicinity of the BH cause the magnetically arrested disk model to resemble to another phenomenological model for the low/hard state, corona-and-disk model. 
The existence of magnetically arrested disks is determined only by the presence or absence of strong vertical magnetic fields. The thermal and emission properties of plasma in these disks are not relevant in this respect. Therefore, magnetically arrested disks can exist in a wide range of luminosities and accretion rates, from significantly sub- to significantly super-Eddington rates. This is unlike the case of optically thin ADAF models that become thermally unstable at moderate and high luminosities, $L \gtrsim 10^{-3} L_{\text {Edd }}$ (Abramowicz et al. 1995).

The magnetically arrested disk model can be used to explain low-frequency $(\sim 0.1-30$ $\mathrm{Hz}$ ) QPOs that are often found in BH binaries in the low/hard state (RM06). The spiral accretion streams in magnetically arrested disks form patterns that have almost solid-body rotations with frequencies determined as a fraction $(\sim 0.5)$ of the Keplerian frequency at $R_{\mathrm{m}}$ (I08). Relating this rotation with low-frequency QPOs, one can estimate the QPO frequency,

$$
\nu \sim 10^{4}\left(\frac{M}{M_{\odot}}\right)^{-1}\left(\frac{R_{\mathrm{m}}}{R_{g}}\right)^{-3 / 2} \mathrm{~Hz} .
$$

Note that Tagger \& Pellat (1999) and Tagger et al. (2004) proposed a similar mechanism of the solid-body rotating structure for low-frequency QPOs. However, the nature of their structure, which is formed by a spiral-wave generated because of the "accretion-injection" instability, is different from the nature of magnetically arrested disks. Estimating $R_{\mathrm{m}}$ from eq. (2) in the case of Cyg X-1, which is characterized by $M \simeq 10 M_{\odot}$ and $\nu \sim 1-3$ $\mathrm{Hz}$ (Rutledge et al. 1999), one obtains $R_{\mathrm{m}} \sim 30 R_{g}$. The latter estimate agrees with the estimates of $R_{\mathrm{tr}}$ in Cyg X-1 obtained from interpretations of X-ray spectra (Gierliński et al. 1997; Axelsson et al. 2008).

\section{Transition Mechanism}

The flow that feeds an accretion disk orbiting the primary $(\mathrm{BH})$ is supplied by the secondary (star) and, therefore, can carry a magnetic field, whose strength and topology is determined by the strength and topology of the stellar magnetic field and relative orbital motions of binary components. In this circumstance, the poloidal component of the supplied fields can be inverted in time randomly (depending on the secondary's magnetic activity) and/or quasi-periodically (with the time scale $\sim$ the orbital time). The field inversion can result in a temporal disappearance of a magnetically arrested disk, because of the annihilation of opposed fields, and in a restoration of an optically thick disk that extends down to the last stable circular orbit of the BH. This can explain the transition between the low/hard state, which is characterized by the presence of a magnetically arrested disk, and the high/soft state, which is the manifestation of an untruncated Shakura-Sunyaev-type disk. 
The minimum magnetic flux that is required to form a magnetically arrested disk can be estimated using equation (1):

$$
\Phi_{0} \sim \pi R_{g}^{2} B_{0} \sim \pi R_{g}^{2}\left(\frac{\dot{M} c}{\theta R_{g}^{2}}\right)^{1 / 2} \approx 4 \cdot 10^{20} \theta^{-1 / 2}\left(\frac{M}{M_{\odot}}\right)^{3 / 2}\left(\frac{\dot{M}}{\dot{M}_{\text {Edd }}}\right)^{1 / 2} \mathrm{Mw},
$$

where $\theta=H / R_{g}<1, H$ is the disk thickness near the BH horizon, and $\dot{M}_{\text {Edd }}=L_{\text {Edd }} / c^{2}$. For comparison, the magnetic flux in the Crab pulsar is estimated $\sim 10^{25} \mathrm{Mw}$. The flux (3) comes from the outer accretion radius $R_{\mathrm{a}}=2 G M / v_{\mathrm{a}}^{2}$, where it is collected during the time $\tau$, so that

$$
\Phi_{0} \sim B_{\mathrm{a}} R_{\mathrm{a}} v_{\mathrm{a}} \tau
$$

where $B_{\mathrm{a}}$ and $v_{\mathrm{a}}$ are the magnetic induction and accretion velocity at $R_{\mathrm{a}}$, respectively. The estimate of $B_{\mathrm{a}}$, depending on the accumulation time $\tau$ and other parameters of the problem, can be obtained by substituting equation (3) into (4), yielding

$$
B_{\mathrm{a}} \sim 10^{-3} \theta^{-1 / 2}\left(\frac{v_{\mathrm{a}}}{c}\right)\left(\frac{\tau}{\text { year }}\right)^{-1}\left(\frac{M}{M_{\odot}}\right)^{1 / 2}\left(\frac{\dot{M}}{\dot{M}_{\text {Edd }}}\right)^{1 / 2} \mathrm{G} .
$$

Let us consider the example of Cyg X-1 and use the following parameters: $v_{\mathrm{a}} \sim 10^{8} \mathrm{~cm} / \mathrm{s}$, $\tau \sim 1$ year, $\theta \sim 0.1$, and $\dot{M} \sim 0.1 \dot{M}_{\text {Edd }}$. Then, the estimate of the inner field yields $B_{0} \sim 10^{8} \mathrm{G}$. This field magnitude agrees with the magnitude derived from polarimetric observations of Cyg X-1 (Gnedin et al. 2003). The estimate of the outer field yields $B_{\mathrm{a}} \sim$ $10^{-5} \mathrm{G}$. This estimate demonstrates how small the field supplied into the accretion disk can be to initiate the formation of a magnetically arrested disk in Cyg X-1. For comparison, the typical observed stellar fields are $\sim 1-100 \mathrm{G}$.

An evolution of the inverted fields in accretion disks has been studied using axisymmetric 2D MHD simulations. Although these simulations do not correctly reproduce the 3D structure of magnetically arrested disks (I08), they qualitatively correctly model the global evolution of the fields and, therefore, are adequate here. The employed numerical method is described in I08 and the simulation setup is assumed to be the same as in Model B from there, except that the present simulations consider an inversion of the injected field. Figure 1 presents the evolution of magnetic fluxes in the midplane of the model inside the five specific radii: $210 R_{g}\left(=R_{\mathrm{inj}}\right), 100 R_{g}, 50 R_{g}, 25 R_{g}$, and $2 R_{g}\left(=R_{\text {in }}\right)$ (the black, red, green, blue, and magenta curves, respectively). The time is given in units of the orbital time at $R_{\text {inj. }}$. The spikes seen in the magenta curve are due to a cycle accretion in the magnetically arrested region. This cycle accretion is an artifact of the assumed axisymmetry and is not present in 3D simulations (I08). Initially, the magnetic fluxes are gradually increased with time because of the inward advection of the vertical field. The moment of the field inversion 
is chosen at $t=5.1$, which is clearly distinguished as the abrupt jump of the black curve in Fig. 1. Other curves, which correspond to the fluxes at smaller radii, are changed in time with delays and more gradually. The field inversion results in a temporal suppression of the accretion flow at large radii (because more injected mass leaves the computational domain through the outer boundary) and development of a wide radial gap, $\sim R_{\text {out }} / 2$, between the outer edge of the "old" disk and the inner edge of a "new" disk, which carries the inverted field. As the mass accumulated in the old disk is reduced, the accretion rate into the $\mathrm{BH}$ is also reduced. The increased time intervals between the spikes seen at $t \approx 6$ to 7.5 in Fig. 1 correspond to this reduction. At $t \approx 7$, the new disk is fully developed and quickly fills the radial gap, forming a continuous disk. This disk has the vertical fields, which are inverted at some disk radius. Figure 2 represents this stage of evolution, showing the density distribution and magnetic lines in the meridional cross-section of the model at $t=8.52$. The central magnetic bundle is large and the flow is magnetically arrested. The inverted field (see Fig. 2b) closely approaches the bundle, resulting in an intensive reconnection dissipation, which heats plasma in a narrow interface between the opposed fields. At $t \approx 9.5$, the central bundle is completely annihilated and the dense disk extends all the way inward to $R_{\text {in }}$. The simulations show that the accretion into the $\mathrm{BH}$ is continuous, without signs of the cycle accretion, from $t \approx 9$ to 10 . Figure 3 illustrates this evolution stage, showing the model at $t=10.08$. The simulations were finished at $t=10.75$. At this moment, the inverted field builds up another central magnetic bundle, which arrests the accretion flow. It becomes apparent that the further evolution of this model will basically repeat the initial evolution.

The SPL state is similar in some respects to the high/soft state (see Sec. 1). This motivates us to explain the SPL state as a final phase of the more extended period of field annihilation that precedes the high/soft state. Unfortunately, no supporting 3D simulations of this period have been done yet and our discussion is only limited by the following qualitative statements. (1) At the final phase, the radius $R_{\mathrm{m}}$ is small and the optically thick disk can extend deep inside, providing a sizable spectral thermal component. (2) A significant fraction of the disk emission can be provided by the reconnection dissipation of the stored magnetic energy. This can explain the specific steep power law spectral feature. (3) Small $R_{\mathrm{m}}$ means that dense blobs and accretion streams, similar to that found by I08, can be developed in the vicinity of the BH horizon. Orbital motions of such blobs and streams near the last stable circular orbit of the BH can explain high-frequency $(\sim 150-450 \mathrm{~Hz})$ QPOs (e.g., Stella \& Vietri 1999; Abramowicz \& Kluźniak 2003). 


\section{Summary}

This letter proposes a detailed mechanism for emission state transition in BH X-ray binaries. The mechanism is based on a dynamical model of a magnetically arrested disk obtained in recent 3D MHD simulations (I08) that can describe the low/hard state. In this state, the central region of an optically thick accretion disk orbiting the $\mathrm{BH}$ is filled with a strong (equipartition) vertical magnetic field that arrests the flow, resulting in a truncated disk. The accretion, however, is not suppressed in this disk but takes the form of thin spiral streams that penetrate through a highly magnetized, hot, low-density plasma. The spiral flow twists the vertical field and produces powerful Poynting jets. Radiation from the disk is dominated by hard X-rays emitted by the hot plasma and includes a radio component from the jets. The magnetically arrested disk is capable to explain low-frequency QPOs.

The transition from the low/hard to high/soft state occurs when the magnetic field at the outer boundary is inverted. An annihilation of this field, carried inward by the flow, and the central field results in a temporal disappearance of the magnetically arrested disk. For

a moment, the optically thick disk extends inward to the last stable orbit of the $\mathrm{BH}$ and no jets are produced. This represents the high/soft state, which ends as soon as the continuous supply of the inverted field results in the formation of another magnetically arrested disk. The SPL state and associated with it high-frequency QPOs develop in this scenario at the period of intensive annihilation of the inverted fields, which precedes the high/soft state. Quasi-periodic or random inversion of magnetic fields in the accretion flow supplied by the secondary star can explain the observed spectral transitions of BH X-ray binaries. 


\section{REFERENCES}

Abramowicz, M. A., Chen, X., Kato, S., Lasota, J.-P., \& Regev, O. 1995, ApJ, 438, L37

Abramowicz, M. A., \& Kluzńiak, W. 2003, Gen. Relativ. Gravitation, 35, 69

Axelsson, M., Hjalmarsdotter, L., Borgonovo, L., \& Larsson, S. 2008, A\&A, 490, 253

Bisnovatyi-Kogan, G. S., \& Ruzmaikin, A. A. 1974, Ap\&SS, 28, 45

Bisnovatyi-Kogan, G. S., \& Ruzmaikin, A. A. 1976, Ap\&SS, 42, 401

Blandford, R. D., \& Begelman M. C. 1999, MNRAS, 303, L1

Cabral, B., \& Leedom, L. 1993, Computer Graphics: Proceedings: Annual Conference Series 1993: SIGGRAPH 93 (New York: Association for Computing Machinery), 263

D’Angelo, C., Giannios, D., Dullemond, C., \& Spruit, H. 2008, A\&A, 488, 441

Done, C., \& Gierliski, M. 2003, MNRAS, 342, 1041

Done, C., Gierliński, M., \& Kubota, A. 2007, A\&A Rev., 15, 1

Dullemond, C. P., \& Spruit, H. C. 2005, A\&A, 434, 415

Esin, A. A., McClintock, J. E., \& Narayan, R. 1997, ApJ, 489, 865

Esin, A. A., Narayan, R., Cui, W., Grove, J. E., \& Zhang, S.-N. 1998, ApJ, 505, 854

Esin, A. A., McClintock, J. E., Drake, J. J., Garcia, M. R., Haswell, C. A., Hynes, R. I., \& Muno, M. P. 2001, ApJ, 555, 483

Ferreira, J., Petrucci, P.-O., Henri, G.. Saugé, L., \& Pelletier, G. 2006, A\&A, 447, 813

Galeev, A. A., Rosner, R., \& Vaiana, G. S. 1979, ApJ, 229, 318

Gallo, E., Fender, R. P., \& Pooley, G. G. 2003, MNRAS, 344, 60

Gierliński, M., Zdziarski, A. A., Done, C., Johnson, W. N., Ebisawa, K., Ueda, Y., Haardt, F., \& Phlips, B. F. 1997, MNRAS, 288, 958

Gnedin, Yu. N., Borisov, N. V., Natsvlishvili, T. M., Piotrovich, M. Yu., \& Silant'ev, N. A. 2003, arXiv: astro-ph/0304158

Haardt, F., Maraschi, L., \& Ghisellini, G. 1994, ApJ, 432, L95 
Honma, F. 1996, PASJ, 48, 77

Igumenshchev, I. V., Narayan, R., \& Abramowicz, M. A. 2003, ApJ, 592, 1042 (INA03)

Igumenshchev, I. V. 2008, ApJ, 677, 317 (I08)

Kubota, A., Ebisawa, K., Makishima, K., \& Nakazawa, K. 2005, ApJ, 631, 1062

Liang, E. P. T., \& Price, R. H. 1977, ApJ, 218, 247

Livio, M., Pringle, J. E., \& King, A. R. 2003, ApJ, 593, 184

Manmoto, T., \& Kato, S. 2000, ApJ, 538, 295

Markoff, S., Falcke, H., \& Fender, R. 2001, A\&A, 372, L25

Merloni, A., \& Fabian, A. C. 2001, MNRAS, 321, 549

Meyer, F., Liu, B. F., \& Meyer-Hofmeister, E. 2000, A\&A, 361, 175

Narayan, R., Yi, I. 1994, ApJ, 428, L13

Narayan, R., Igumenshchev, I. V., \& Abramowicz, M. A. 2000, ApJ, 539, 798

Narayan, R., Igumenshchev, I. V., \& Abramowicz, M. A. 2003, PASJ, 55, L69

Petrucci, P. O., Ferreira, J., Henri, G., Sauge, L., \& Pelletier, G. 2006, Advances in Space Research, 38, 2909

Poutanen J., Krolik J. H., \& Ryde F. 1997, MNRAS, 292, L21

Punsly, B., \& Coroniti, F. V. 1990, ApJ, 354, 583

Punsly, B., Hirose, S., \& Igumenshchev, I. V. 2009, ApJ, submitted

Remillard, R. A., \& McClintock, J. E. 2006, ARA\&A, 44, 49 (RM06)

Rutledge, R. E., Lewin, W. H. G., van der Klis, M., van Paradijs, J., Dotani, T., Vaughan, B., Belloni, T., Oosterbroek, T., \& Kouveliotou, C. ApJS, 124, 265

Shakura, N. I., \& Sunyaev, R.A. 1973, A\&A, 24, 337

Shapiro, S. L., Lightman, A. P., \& Eardley, D. M. 1976, ApJ, 204, 187

Spruit, H. C., \& Deufel, B. 2002, A\&A, 387, 918

Stella, L., \& Vietri, M. 1999, Phys. Rev. Let., 82, 17 
Taam, R. E., Liu, B. F., Meyer, F., \& Meyer-Hofmeister, E. 2008, ApJ, 688, 527

Tagger, M., \& Pellat, R. 1999, A\&A, 349, 1003

Tagger, M., Varniére, P., Rodriguez, J., \& Pellat, R. 2004, ApJ, 607, 410

Uzdensky, A. A., \& Goodman, J. 2008, ApJ, 682, 608

Zdziarski, A. A., \& Gierliński, M. 2004, Prog. Theor. Phys. Suppl., 155, 99 
Fig. 1.- Evolution of vertical magnetic fluxes (in arbitrary units) in axisymmetric 2D simulations of the accretion disk model. The five curves shown correspond to the fluxes through the equatorial plane inside five fixed radii: $210 R_{g}$ (= $R_{\mathrm{inj}}$, black), $100 R_{g}$ (red), $50 R_{g}$ (green), $25 R_{g}$ (blue), and $2 R_{g}$ (magenta). The time is in units of the orbital time at $R_{\text {inj }}$. The injected field is inverted at $t=5.1$.

Fig. 2.-Distribution of density (a) and magnetic lines (b) in the meridional plane at $t=8.52$ from axisymmetric 2D simulations of the accretion disk model. The black hole is located on the left side of the images, and the small open circles there correspond to the inner boundary around the black hole at $R_{\text {in }}=2 R_{g}$. The axis of rotation is in the vertical direction. The domain shown has the radial extend $R_{\text {out }}=220 R_{g}$ along the equatorial plane and vertical extend from $-R_{\text {out }} / 2$ to $R_{\text {out }} / 2$. The color bar on the right in (a) indicates the scale for $\log \rho$ (in arbitrary units). The lines in (b) have been plotted using the method of Cabral \& Leedom (1993). The field carried inward by the disk and the field in the central magnetic bundle have inverted vertical components (b). The central bundle arrests the accretion flow and forms a truncated disk (a).

Fig. 3.- Same as in Figure 2, but at $t=10.08$. The central magnetic bundle seen in Figure $2 \mathrm{~b}$ has disappeared because of the annihilation with the incoming inverted field (b). The disk is untruncated and extends all the way inward to $R_{\text {in }}(\mathrm{a})$. 
This figure "fig1.png" is available in "png" format from: http://arxiv.org/ps/0908.0431v1 
This figure "fig2a.png" is available in "png" format from: http://arxiv.org/ps/0908.0431v1 
This figure "fig2b.png" is available in "png" format from: http://arxiv.org/ps/0908.0431v1 
This figure "fig3a.png" is available in "png" format from: http://arxiv.org/ps/0908.0431v1 
This figure "fig3b.png" is available in "png" format from: http://arxiv.org/ps/0908.0431v1 